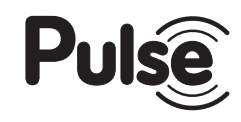

\title{
Various Indices of Arterial Stiffness: Are They Closely Related or Distinctly Different?
}

\author{
Hirofumi Tanaka \\ Cardiovascular Aging Research Laboratory, Department of Kinesiology and Health \\ Education, The University of Texas at Austin, Austin, TX, USA
}

\section{Keywords}

Arterial elasticity · Arteriosclerosis · Methodology · Vascular function

\begin{abstract}
Arterial stiffness is an independent risk factor for cardiovascular disease. There are a number of techniques and devices that have been developed and utilized to capture the information pertaining to the elasticity of the blood vessel. Almost all the available indices of arterial stiffness are known to increase with advancing age and are elevated in the presence of hypertension and coronary heart disease. It is not known how closely these different measures of arterial stiffness are related to each other. Available evidence indicates that arterial stiffness indices that share a homogeneous methodology appear to demonstrate good correlations. However, there are no significant associations between some measures. These overall results may be surprising considering that all the indices are supposed to reflect the same property of the arterial wall (i.e., arterial elasticity). Interestingly, no or weak correlations between indices of vascular function are not confined only to arterial stiffness and can be extended to endothelial function and vascular reactivity measures.

(c) 2017 S. Karger AG, Basel
\end{abstract}

\section{Arterial Stiffness Methodologies}

Arterial stiffness is being recognized as an effective prognostic marker as well as a therapeutic target. Because arterial stiffening is one of the earliest detectable changes in vascular structure and function that lead to clinically apparent and overt vascular diseases, a chase for the easy, precise, reliable, and optimum measurement of arterial stiffness has been intensified [1-3]. As a result, a number of techniques and devices have been developed to capture

Hirofumi Tanaka, PhD

Department of Kinesiology and Health Education

The University of Texas at Austin

2109 San Jacinto Blvd., D3700, Austin, TX 78712 (USA)

E-Mail hatanaka@austin.utexas.edu 
Fig. 1. Various measures of arterial stiffness.

Tanaka et al.: Various Indices of Arterial Stiffness: Are They Closely Related or Distinctly Different?

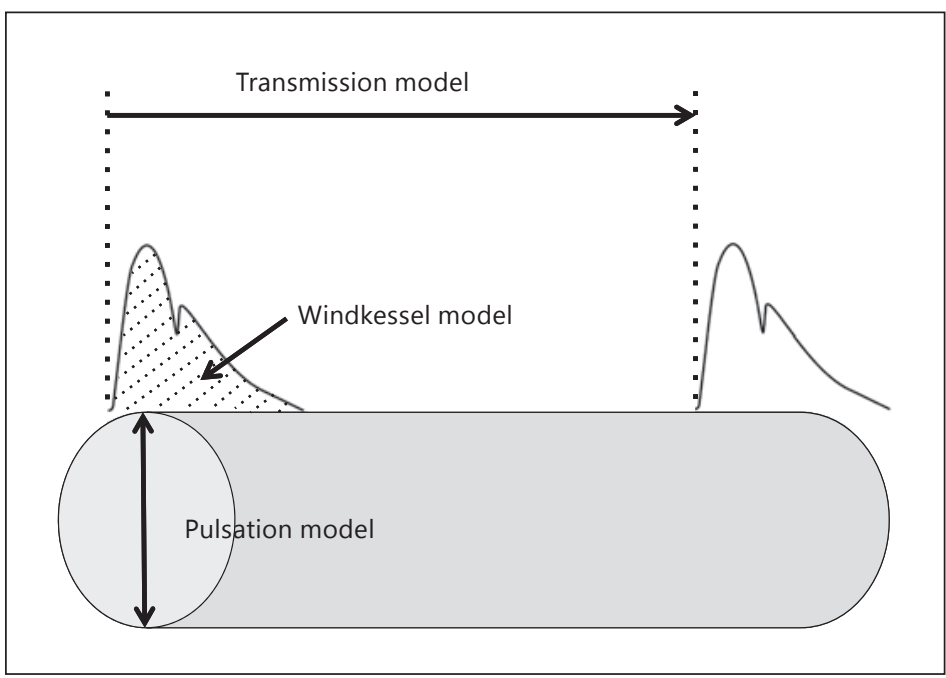

the information pertaining to the elasticity of the blood vessel, and many of these devices are currently available on the commercial market. Most of these methodological approaches to arterial stiffness can be classified into 1 of the following 3 models as shown in Figure 1:

- Transmission or propagation model (e.g., various pulse wave velocity [PWV] measures).

- Pulsation or distension model (e.g., ultrasound-derived carotid artery compliance and distensibility).

- Windkessel model (e.g., C1 and C2 measured with a HDI device, systemic arterial compliance via area method).

Currently, there is no consensus as to which method of arterial stiffness should serve as the "gold standard" with which to compare other measures, although carotid-femoral PWV (cfPWV) has been proposed as the "reference standard" measure of arterial stiffness because of the accumulated and the most abundant clinical evidence linking it to increased mortality [4]. However, cfPWV is an indirect measure to estimate arterial stiffness using mathematical equations and involves a number of assumptions (e.g., constant blood viscosity) [5]. Accordingly, it cannot be considered a gold standard. Almost all the available indices of arterial stiffness are known to increase with advancing age and are elevated in the presence of hypertension and coronary heart disease. The central question that is posed in the present minireview is how closely these different measures of arterial stiffness are related to each other or whether these methodologies are distinctly different. The information will be important for the purpose of interstudy comparisons of arterial stiffness.

\section{Interrelations of Arterial Stiffness Indices}

Recently, we performed comparisons of multiple indices of arterial stiffness within the same study population [6]. Arterial stiffness indices examined included cfPWV, brachialankle PWV (Omron Healthcare), cardio-ankle vascular index (CAVI; Fukuda Denshi), carotid artery compliance (simultaneous application of ultrasound and arterial applanation tonometry), elastic modulus, arterial distensibility, $\beta$-stiffness index, and Young's elastic modulus. To address the interrelationships as comprehensively as possible, we used 3 different but complementary approaches to conduct comparisons between different indices of arterial stiffness [6]. First, we used simple correlational analyses to evaluate associations 
Tanaka et al.: Various Indices of Arterial Stiffness: Are They Closely Related or Distinctly Different?

Table 1. Interrelationships between different measures of arterial stiffness indices at baseline

\begin{tabular}{llllllll}
\hline & cfPWV & baPWV & Compli & ElaMod & Distens & $\beta$-Stiff & Young \\
\hline CAVI & 0.74 & 0.82 & -0.38 & 0.45 & -0.29 & ns & ns \\
cfPWV & - & 0.69 & -0.39 & 0.47 & -0.31 & ns & ns \\
baPWV & & - & -0.44 & 0.54 & -0.32 & ns & ns \\
Compli & & & - & -0.96 & 0.96 & -0.91 & 0.82 \\
ElaMod & & & - & -0.86 & 0.79 & 0.76 \\
Distens & & & & & - & -0.96 & 0.83 \\
B-Stiff & & & & & & - & 0.81
\end{tabular}

Data are Pearson correlation coefficients. CAVI, cardio-ankle vascular index; cfPWV, carotid-femoral pulse wave velocity; baPWV, brachial-ankle pulse wave velocity; Compli, arterial compliance; ElaMod, elastic modulus; Distens, arterial distensibility; $\beta$-Stiff, $\beta$-stiffness index; Young, Young's modulus; ns, not significant. Reproduced with permission from the publisher [6].

between basal measures of arterial stiffness. Second, a combination of Bland-Altman plots with calculations of $95 \%$ confidence intervals was utilized to evaluate the limit of agreements between measures. Third, relative changes in arterial stiffness indices in response to isometric exercise were compared. The consistent findings across all 3 approaches were that measures of arterial stiffness were variably associated with each other, ranging from no significant relations to highly significant associations (Table 1). Arterial stiffness indices that share a homogeneous methodology appear to demonstrate stronger correlations. However, there are no significant associations between some measures [6]. More specifically, cfPWV was associated with other transmission or propagative measures of arterial stiffness, including brachialankle PWV and CAVI, but the associations of cfPWV with ultrasound-derived measures of arterial compliance were very mild. Although all measures of ultrasound-based arterial stiffness were derived from the same measurements of arterial diameter and local pressure through a variety of mathematical equations, the associations between them were not strong, suggesting that each measure may attempt to reflect some unique aspects of the arterial wall properties [6].

These results are not isolated findings and are in agreement with other research. For example, poor agreements have been observed between cfPWV and measures derived from the Windkessel model (i.e., C1 and C2, systemic arterial compliance) [7]. Even within the techniques for the measurement of cfPWV in the same population, there is a substantial difference in cfPWV values measured with SphygmoCor and Complior presumably due to different algorithms to detect the foot of pressure pulses required in PWV measurements [8].

As shown in Figure 1, measures of arterial stiffness can be divided into 3 primary methodological models. The transmission or propagation model (e.g., PWV) involves "regional" measurements that track movements of pulse waves from one location to another along the arterial tree, whereas the pulsation model (e.g., carotid arterial compliance) typically consists of "local" assessments in that the ultrasound transducer is placed on one artery. Some measures (e.g., $\beta$-stiffness index and CAVI) claim to be blood pressure independent, whereas others are highly dependent on blood pressure [9]. Physiological/hemodynamic determinants and strengths of correlations with various risk factors for cardiovascular disease appear to be very variable among arterial stiffness indices [10]. Moreover, arterial stiffness varies widely depending on the location of the measurements. Even within the same cardiothoracic arteries, the aorta and carotid arteries appear to undergo different degrees of atherosclerotic and arteriosclerotic processes [11]. Considering these differences in many aspects involved 
in arterial stiffness measurements, it might be reasonable to expect variable degrees of associations between arterial stiffness indices.

However, for a number of people, these overall results may surely be surprising considering that all the indices are supposed to reflect the same property of the arterial wall (i.e., arterial elasticity). How should these results be interpreted? If one sees the glass as half full, this could suggest that some measures of arterial stiffness may provide incrementally useful information that is not given by one methodology only and that these measures combined would provide additive values for a more comprehensive and more complete assessment of vascular function. On the other hand, if one sees the glass as half empty, this would suggest that the findings obtained by one methodology cannot be extrapolated to those obtained by different techniques and that the research field of arterial stiffness may be disordered and disorganized.

\section{Blood Pressure Dependence of Arterial Stiffness Measures}

Elevated arterial stiffness mechanically leads to increased systolic blood pressure and pulse pressure. Conversely, elevated blood pressure affects the measurements of arterial stiffness to varying degrees. Indeed, blood pressure dependence of arterial stiffness has been a topic of much discussion among investigators working in the field. Some have questioned the utility of arterial stiffness above and beyond the transitional brachial blood pressure measurements, as changes in arterial stiffness are often accompanied by the corresponding changes in blood pressure. In order to gain further insight into this blood pressure dependency, we recently assessed the effects of acute blood pressure perturbations on various measures of arterial stiffness [9]. Because there are a number of blood pressure perturbation maneuvers that utilize different forms of stress (psychological, physical, and mechanical), we used a total of 5 different maneuvers, including head-up tilt, head-down tilt, mental stress, isometric handgrip exercise, and cold pressor test. Each test produced different magnitudes of pressor responses. In conjunction with the blood pressure changes, corresponding changes in most of the arterial stiffness indices were observed during each maneuver. Thus, most, if not all, of the arterial stiffness indices displayed some degree of blood pressure dependency on various forms of pressor tasks. This was even true for indices of arterial stiffness that are thought to be or claimed to be blood pressure independent (e.g., $\beta$-stiffness index and CAVI). More importantly, the strengths of associations with blood pressure varied widely depending on which arterial stiffness indices were examined [9]. These results are consistent with the notion that various measures of arterial stiffness assess different elements in the arterial wall that are differently affected by blood pressure changes.

\section{Interrelations of Other Vascular Function Measures}

Do we observe similar methodological differences in other measures of vascular function? The augmentation index was used in the past as a measure of arterial stiffness. But in more recent years, it has been considered an index of arterial wave reflection that is indirectly associated with arterial stiffness. There are a number of commercially available devices that attempt to measure this property. One previous study from our laboratory assessed associations of augmentation indices assessed in the finger (Itamar), radial artery (Omron Healthcare), and aorta (via transfer function; SphygmoCor). The correlation coefficients ranged from 0.78 to 0.94 [12]. These associations are fairly strong considering that these techniques assess augmentation indices in different vascular beds using varying techniques and conversions. 
Another important measure of vascular function is endothelial function. Similar to arterial stiffness, many methodological approaches have been developed to assess physiological vasodilatory capacity of the endothelium in humans. Because pharmacological infusion of vasoactive drugs into the coronary artery is highly invasive, most of these new techniques assess endothelial function in peripheral arteries (e.g., brachial artery) that are more easily accessible. One of the commonly used invasive methods in the research setting is to utilize intrabrachial infusion of vasoactive drugs, such as acetylcholine or bradykinin, combined with venous occlusion plethysmography to assess subsequent changes in forearm blood flow [13]. More commonly, endothelial function can be measured noninvasively and physiologically using flow-mediated dilation (FMD). In the flow-mediated dilation technique, reactive hyperemia following a 5-min occlusion of the brachial artery with a blood pressure cuff triggers endothelial NO release and causes brachial artery diameter (measured with the ultrasound machine) to increase. Both of these methodologies have been found to be mainly NO dependent in pharmacological blockade experiments. However, we found that these measures of endothelial function were not significantly associated with each other when both methods were performed in the same population [14]. Recently, a number of noninvasive alternative approaches to evaluate vascular reactivity following brachial ischemia have been introduced. These techniques monitor various physiological signals in an attempt to assess endothelial function. We compared a total of 7 different measures of vascular reactivity in the same subjects [15]. They included flow-mediated dilation, hyperemic shear stress, reactive hyperemic flow measured with ultrasound, changes in PWV between the brachial and radial artery, reactive hyperemia index assessed by fingertip arterial tonometry (EndoPat), fingertip temperature rebound (Endothelix), and skin-reactive hyperemia measured with a laser Doppler monitor. The results indicated that measures of vascular reactivity were not strongly associated with each other. In most of the techniques assessed, correlations between vascular reactivity measures did not even reach statistical significance [15]. Collectively, these results suggest that varying degrees of physiological mechanisms (in addition to NO) may be involved in evoking different peripheral vascular responses. Thus, no or weak correlations between indices of vascular function are not confined to arterial stiffness only and can be extended to endothelial function and vascular reactivity measures.

\section{Disclosure Statement}

H. Tanaka serves in the scientific advisory board of Endothelix. There is no other conflict of interest to be disclosed.

\section{References}

1 Cortez-Cooper MY, Supak JA, Tanaka H: A new device for automatic measurements of arterial stiffness and ankle-brachial index. Am J Cardiol 2003;91:1519-1522.

2 Sugawara J, Tanaka H: Brachial-ankle pulse wave velocity: myths, misconceptions, and realities. Pulse (Basel) 2015;3:106-113.

3 Hartley CJ, Tanaka H: Assessment of macro- and microvascular function and reactivity; in Naghavi M (ed): Asymptomatic Atherosclerosis: Pathophysiology, Detection and Treatment. New York, NY, Humana Press, 2011, pp 265-278.

4 Van Bortel LM, Laurent S, Boutouyrie P, et al: Expert consensus document on the measurement of aortic stiffness in daily practice using carotid-femoral pulse wave velocity. J Hypertens 2012;30:445-448.

5 Bramwell JC, Hill AV: The velocity of the pulse wave in man. Proc R Soc Lond B Biol Sci 1922;93:298-306.

6 Lim J, Pearman M, Park W, Alkatan M, Tanaka H: Interrelationships among various measures of central artery stiffness. Am J Hypertens 2016;29:1024-1028.

7 Woodman RJ, Kingwell BA, Beilin LJ, Hamilton SE, Dart AM, Watts GF: Assessment of central and peripheral arterial stiffness: studies indicating the need to use a combination of techniques. Am J Hypertens 2005;18: 249-260. 
8 Millasseau SC, Stewart AD, Patel SJ, Redwood SR, Chowienczyk PJ: Evaluation of carotid-femoral pulse wave velocity: influence of timing algorithm and heart rate. Hypertension 2005;45:222-226.

9 Lim J, Pearman ME, Park W, Alkatan M, Machin DR, Tanaka H: Impact of blood pressure perturbations on arterial stiffness. Am J Physiol Regul Integr Comp Physiol 2015;309:R1540-R1545.

10 Tanaka H, Dinenno FA, Hunt BE, Jones PP, DeSouza CA, Seals DR: Hemodynamic sequelae of age-related increases in arterial stiffness in healthy humans. Am J Cardiol 1998;82:1152-1155.

11 van Popele NM, Grobbee DE, Bots ML, et al: Association between arterial stiffness and atherosclerosis: the Rotterdam Study. Stroke 2001;32:454-460.

12 Dhindsa M, Barnes JN, Devan AE, Sugawara J, Tanaka H: Comparison of augmentation index derived from multiple devices. Artery Res 2011;5:112-114.

13 DeSouza CA, Shapiro LF, Clevenger CM, et al: Regular aerobic exercise prevents and restores age-related declines in endothelium-dependent vasodilation in healthy men. Circulation 2000;102:1351-1357.

14 Eskurza I, Seals DR, DeSouza CA, Tanaka H: Pharmacological vs. flow-mediated assessments of peripheral vascular endothelial vasodilatory function in humans. Am J Cardiol 2001;88:47-49.

15 Dhindsa M, Sommerlad SM, Devan AE, et al: Interrelationships among noninvasive measures of postischemic macro- and microvascular reactivity. J Appl Physiol 2008;105:427-432. 